\title{
Aetiological factors in infection following open retrograde intramedullary nailing of the femur
}

\author{
Abulfotooh M. EID \\ M.Ch. Orth.
}

\author{
P.O. Box 42, Doha, Qatar, Arabian Gulf
}

\begin{abstract}
Summary
Thirty-three patients with chronic osteomyelitis of the femur following open retrograde Küntscher intramedullary nailing of the femur were studied to determine possible reasons for the development of infection. In 32 patients the indication for the operation was a closed fracture of the shaft of the femur (recent or malunited) and in one it was to shorten the femur. In 6 patients $(18.2 \%)$ there was evidence of skin, urinary and upper respiratory tract infection. In 14 patients $(42.4 \%)$ it was felt that potential infection was present in poor skin overlying the fracture and unhealed laparotomy wounds. Four patients were under the age of 15 years. The operating theatres lacked facilities for room-air exchange, for provision of filtered air or for modern body-exhaust systems. Twenty-two (66.7\%) operations were performed by surgeons under training.
\end{abstract}

\section{Introduction}

There are obvious advantages in the treatment of closed fractures of the shaft of the femur by intramedullary nailing; these are outweighed if the incidence of infection is high, as the consequences are serious. A $29 \%$ amputation rate has been reported by Weber and Hirtzman (1964), and the economic loss to the patient may be considerable.

In this study the possible reasons for infection after the insertion of the intramedullary nail by the open retrograde procedure for closed fractures of the shaft of the femur were investigated in retrospect so that precautions might be taken to avoid their occurrence in the future.

\section{Material}

Thirty-three patients suffering from chronic osteomyelitis of the femur following open retrograde Küntscher intramedullary nailing of the femur were examined and treated by the author in the years 1965-1977 inclusive. Twenty-four were male. Their ages ranged from 9 to 78 years; 4 were $<15$ years old. The indication for operation was a closed fracture of the shaft of the femur in 32 , and in one it was to shorten the femur. The fracture was in the upper third of the femur in 7 patients, in the middle third in 10 , and in the lower third in 16.

In 21 patients the fractures were recent. These were comminuted in 7 , oblique in 5 and transverse in 9. Multiple skeletal injuries were present in 11, and visceral injuries in 9; laparotomy was required in 7 of the latter. Intramedullary nail insertion was carried out from 4 to 17 days after the injury.

In 11 patients, the operation was performed because of failure of conservative treatment. This included immobilization of a hip spica for 10 weeks in one patient, and skeletal traction for 6 and 10 weeks in 2 patients. In the remaining 8 , the fractures were malunited for some years.

Results

Condition of the patient

Infection: At the time of operation 2 patients had superficial sacral sores, one a splint ring sore, 2 mild bronchitis and one unrecognized urinary tract infection.

Status of the injured limb: In one patient the skin of the affected limb was macerated after immobilization in a hip spica. In another 8 there were unhealed abrasions in the region of the fracture site.

General condition: Seventeen patients $(51.5 \%)$ had a haemoglobin concentration $>60 \%$.

\section{Environmental conditions}

The operating theatres: These had no room-air exchange facilities.

Sterilization: In 19 operations $(57 \cdot 6 \%)$, up-to-date sterilization technique and equipment was not available. In most cases sterilization was carried out by boiling. When pressure sterilization was used, the downward displacement, low-speed autoclave was the apparatus most available.

Instruments and intramedullary nails were mostly of dissimilar nature. No suction drainage was used postoperatively. 
The operation procedure

(a) On 22 occasions $(66.7 \%)$, the operation was carried out by surgeons under training.

(b) Most of the operations lasted an average 90 min. Eleven operations were prolonged for more than $2 \mathrm{hr}$ because of technical difficulties.

(c) Supplementary internal fixation using silk or wire circlage was used in 12 patients $(36.4 \%)$.

\section{Bacteriological investigations}

No organisms were grown from the wounds of 13 cases. In 20 patients, organisms isolated were Staphylococcus aureus in 8; Escherichia coli in 2; Proteus vulgaris, Pseudomonas pyocyanea and Streptococcus pyogenes in one each, and in the remaining 7 patients a mixture of 2 or more pathogens were identified.

\section{Discussion}

The reasons for postoperative infection have been carefully investigated. Endogenous infection may result from organisms derived from septic foci in the patient himself. Exogenous sources of infection include the surgical team, the attendants, the instruments used at operation, the theatre air, or the dressings used later. Both endo- and exogenous sources contributed to the incidence of infection in the present series.

Before operation, possible active infection existed in 6 patients, while in another 14 there were potential sources of infection (unhealed laparotomy wounds and unhealthy skin at the operation site). Twentyone patients $(63.6 \%)$ were at a high risk of infection. Four were children under the age of 15 years, when intramedullary nailing has been considered inadvisable (Böhler, 1948; Burwell, 1971; O’Brien, 1963). Growing tissues have poor resistance to infection as their blood may contain fewer antibodies to the commonly offending pathogens. Seventeen patients were anaemic owing to loss of blood. Wickstrom, Corban and Vise (1968) stressed that surgical (oligaemic) shock lowers the patient's resistance to infection. Multiple skeletal injuries were present in 11 patients. Kovacs, Richard and Miller (1973) reported an $18 \%$ incidence of postoperative infection after internal fixation of the femur associated with multiple skeletal injuries, compared to only $4 \%$ in cases of isolated fracture of the femur. The timing of the operation after injury was also relevant. In this series there were delays of 4 days to 10 weeks. Postoperative infection was rare when intramedullary nailing was performed on the day of the accident even in the presence of skin abrasions (O'Brien, 1963) and its incidence increased in direct proportion to the time which elapsed between injury and operation (Kovacs et al., 1973). Wheeler (1960) found that patients who had recently been in hospital, or had relatives in hospital, were more likely to develop infection. This may be due to the acquisition of hospital strains of bacteria on the $\varnothing$

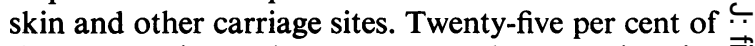
these organisms do not respond to antiseptics $\overrightarrow{\bar{s}}$ (W. Waugh, personal communication). Moreover, $\frac{\overrightarrow{0}}{0}$ the presence of a long-standing haematoma can act as 듬 potential culture medium for organisms. If these $\overline{\bar{\omega}}$ factors had been avoided, the incidence of endogen- $\widehat{\Phi}$ ous postoperative infections would have been greatly reduced or even avoided (Charnley and Eftekhar, क 1969).

The operating theatres may not always be ideal $\vec{\overrightarrow{ }}$ for a procedure of this kind. These patients were $\vec{\omega}^{\circ}$ operated upon in ordinary operating looms with no $\overline{0}$ room-air exchange facilities. Fitzgerald et al. (1977) found that deep sepsis was twice as frequent if $(P<0.05)$ after total hip arthroplasties carried out $\mathrm{G}$ in operating rooms with lower rates of room-air $\stackrel{\infty}{c}$ exchange. The lack of filtered air, body exhaust and air exchange increase the risk of infection. In such $\vartheta$ conditions, excessive movements of the theatre 0 personnel increase air turbulence and aid the settling of dust particles on to the wound. It was $z$ also shown that epithelial squamae from the surgical team carry a high bacterial count and are potentially infective (W. Waugh, personal communication Thomas in 1956 (Report, 1968), found an increase is the count of staphylococci in theatres after operation for the same reasons. The risk of infection becom higher if the operation time is prolonged in such cif cumstances (Stevens, 1964; Tachdjian and Compere, 1957) and essentially if tissue destruction is caused by forceful manipulation and wide dissection. The insertion of an endoprosthesis into the body, alone in- $\mathbb{D}$ creases the liability to infection (Key, 1955). $\overrightarrow{\vec{P}}$ Moreover, in most of the cases, sterilization of the instruments was undertaken by boiling. Boiling does not kill all micro-organisms unless it is continued for many hours (Perkins, 1965) and this was not possible as other operations had to be carried out. In addition, postoperative haematoma formation was not prevented because no suction-drainage was used after any of these operations. These factors must have contributed materially to the occurrence of exogenous postoperative infection. The dissimilar nature of the metals used may result in corrosion reactions with its possible bearing on the incidence of infection.

Luck (1950) stressed that virulent bacteria must be present in large numbers to initiate infection in $\sigma$ bone. Experimental work has shown that there must $N$ also be local tissue devitalization. Intravenous or $\mathrm{N}$ even intra-osseous injection of staphylococci failed to produce osteomyelitis except after local injection of the metaphysis with sodium morrhuate (Scheman et al., 1943). The resulting necrosis allowed infec- on tion to become established. In clinical practice 0 
unfavourable conditions may create such an atmosphere. The denuding of the bone-ends for a distance of 1-10 mm on either side of their blood supply (Böhler, 1948) along with comminution and the wide displacement of the fragments result in cell death. Prolonged exposure and forceful manipulation increase the damage. Pieces of subcutaneous fat left in the operation field were avascular and, in association with haematoma formation, these act as a potential focus for infection; suction drainage was not employed in the present series.

It is clear that in the patients studied in this series many of the factors which contribute to postoperative infection were present. Avoidance of these factors should reduce the incidence of infection.

Ayliffe et al. (1969) believe that most infections encountered are due to a 'personal factor' including lapses in technique from ignorance, overwork, and fatigue. Another important factor is the reliance on antibiotics and the resulting deterioration in aseptic techniques. It has been shown that prophylactic antibiotics not only fail to prevent postoperative infection (Karl et al., 1966) but they may even increase the risk of infection when administered systemically (Derian and Green, 1966) or locally (Miller, 1963).

Watson Cheyne in 1894 stated that suppuration occurring in a wound made by a surgeon through unbroken skin was due to some oversight on the part of the surgeon. It is evident from this study that Watson Cheyne's observations are still largely correct.

\section{Conclusions}

1. The insertion of an intramedullary nail for fracture of the shaft of the femur must be carried out or supervised by a surgeon experienced in the technique of intramedullary nailing. This will reduce the operation time and any unnecessary manoeuvre.

2. Infection, active or potential, must be energetically treated before operation. Haematoma formation must be prevented by suction drainage.

3. Theatres must be surgically sterile and theatre disciplines must be strict.

4. The operation must be avoided in young children.

\section{References}

Ayliffe, G.A.J., Brightwell, K.M., Collins, B.J. \& LOWBURY, E.J.L. (1969) Varieties of aseptic practice in hospital wards. Lancet, ii, 1117.
BoHLER, L. (1948) Medullary Nailing of Küntscher. 1st English ed, translated by Hans Trettor. London, Baillière, Tindall and Cox.

BURWELL, H.N. (1971) Internal fixation in the treatment of fractures of the femoral shaft. Injury, 2, 235.

Charnley, J. \& Eftekhar, N. (1969) Post-operative infection in prosthetic replacement of the hip joint. British Journal of Surgery, 56, 641 .

CheYne, W. (1894) Quoted by Waugh, W., 1972.

Derian, P.S. \& Green, B.M. (1966) Post-operative wound infection. Five years' review of 1163 consecutive operative orthopedic patients. American Surgery, 32, 388.

Fitzgerald, H.J.R., Nolan, D.R., Ilstrup, D.M., Scoy, R.E., WAShington II, I.A. \& Coventry, M. (1977) Deep wound sepsis following total hip arthroplasty. Journal of Bone and Joint Surgery, 59A, 847.

Karl, R.C., Mertz, J.J., Veith, F.J. \& Dineen, P. (1966) Prophylactic antimicrobial drugs in surgery. New England Journal of Medicine, 275, 305.

KEY, J.A. (1955) Indications and contra-indications for medullary nailing of fractures. Journal of the American Medical Association, 158, 1001.

Kovacs, A.J., RichaRd, L.B. \& Miller, J. (1973) Infection complicating intramedullary nailing of the fractured femur. Clinical Orthopaedics, 96, 266.

LucK, V.J. (1950) Acute infections of bones and joints and chronic osteomylitis. In: Bone and Joint Diseases, 1st ed, p. 43. Charles C. Thomas, Springfield, Ill.

Miller, W.E. (1963) Local antibiotic installation in orthopedic surgery. Journal of Bone and Joint Surgery, 45A, 433.

O'Brien, J.P. (1963) Fractured femoral shafts. A review of 127 consecutive cases including 53 treated by Küntscher nail fixation. Australian and New Zealand Journal of Surgery, 33, 91.

PERKINS, E.W. (1965) Aseptic Technique for Operating Room Personnel. 2nd ed, p. 32. W. B. Saunders Co., Philadelphia and London.

REPORT (1968) Aseptic methods in the operating suite. Report to the Medical Research Council by the Subcommittee on aseptic methods in operating theatres of their Committee on hospital infection. Lancet, i, 705, 763, 831.

Scheman, L., Lewin, R., Sediman, S. \& Janota, M. (1943) Experimental osteomyelitis. American Surgery, 60, 371.

Stevens, D.B. (1964) Post-operative orthopedic infections. A study of the etiological factors. Journal of Bone and Joint Surgery, 46A, 96.

TAChDJiAn, M.O. \& Compere, E.L. (1957) Post-operative wound infections in orthopedic surgery. Evaluation of prophylactic antibiotics. Journal of the International College of Surgeons, 28, 797.

Thomas, C. (1956) Quoted in Report, 1968.

Weber, R. \& HiRtzman, I. (1964) Les ostéites de fémur après enclouage centremédullaire. Revue de Chirurgie orthopédique et réparatrice de l'appareil moteur, 50, 77.

WHEELER, W.E. (1960) Certain aspects of the treatment of staphylococcal infections. Pediatric Clinics of North America, 7, 825.

Wickstrom, J., Corban, M.A. \& Vise, G.T. (1968) Complications following intramedullary fixation of 324 fractured femurs. Clinical Orthopaedics. Philadelphia, London, 60, 103. 